\title{
Hybrid evolution strategy approach for robust permutation flowshop scheduling
}

\author{
Khurshid, B. ${ }^{a}$, Maqsood, S. ${ }^{\text {b, }}$, Omair, M. ${ }^{b}$, Nawaz, R. ${ }^{a}$, Akhtar, R. ${ }^{a}$ \\ ${ }^{a}$ Department of Industrial Engineering, University of Engineering and Technology, Peshawar, Pakistan \\ ${ }^{b}$ Department of Industrial Engineering, Jalozai Campus, University of Engineering and Technology, Peshawar, Pakistan
}

\begin{abstract}
A B S T R A C T
In this paper, a robust schedule has been proposed to deal with uncertainities for $m$-machines permutation flow shop problems. A robust schedule ensures that the expected finish time is always less than the makespan. To use the global search ability of the evolution strategy (ES) and local search ability of Tabu Search (TS), a hybrid evolution strategy (HES) is proposed by combining Improved ES with TS to generate the robust schedules. The robust schedule is first generated using ES and then the solution is optimized using TS for maximum exploitation and exploration of the solution space. For maximum exploitation in ES, (1+9) reproduction operator and double swap mutation is used. Also variable mutation rate is used for fine tuning of the results. In TS, the length of Tabu list is fixed, also lower bound is used to save computational time. The hybrid algorithm is tested on Carlier and Reeves benchmark problems taken from the OR-library. Achieved results are compared with other famous techniques available in the literature, and the results show that HES performs better than other techniques and provides an affirmative percentage increase in the probability that the expected finish time is less than the makespan.
\end{abstract}

(C) 2020 CPE, University of Maribor. All rights reserved.

\begin{tabular}{l} 
A R T I C L E I N F O \\
\hline Keywords: \\
Permutation flowshop; \\
Scheduling; \\
Carlier problem; \\
Reeves problem; \\
Evolutionary computation; \\
Hybrid evolution stratgey; \\
Improved evolution strategy; \\
Tabu search \\
*Corresponding author: \\
smaqsood@uetpeshawar.edu.pk \\
(Maqsood, S.) \\
Article history: \\
Received 1 April 2020 \\
Revised 12 July 2020 \\
Accepted 14 July 2020
\end{tabular}

\section{References}

[1] Pinedo, M.L. (2015). Scheduling, Theory, Algorithms, and Systems, Fifth Edition, Springer, New York, USA, doi: 10.1007/978-3-319-26580-3.

[2] Sturrock, D. (2012). New solutions for production dilemmas, Industrial Engineer, Vol. 44, No. 12, 47-52.

[3] Gourgand, M., Grangeon, N., Norre, S. (2003). A contribution to the stochastic flow shop scheduling problem, European Journal of Operational Research, Vol. 151, No. 2, 415-433, doi: 10.1016/S0377-2217(02)00835-4.

[4] Juan, A.A., Barrios, B.B., Vallada, E., Riera, D., Jorba, J. (2014). A simheuristic algorithm for solving the permutation flow shop problem with stochastic processing times, Simulation Modelling Practice and Theory, Vol. 46, 101-117, doi: 10.1016/i.simpat.2014.02.005.

[5] Elyasi, A., Salmasi, N. (2013). Stochastic flow-shop scheduling with minimizing the expected number of tardy jobs, The International Journal of Advanced Manufacturing Technology, Vol. 66, No. 1-4, 337-346, doi: 10.1007/ s00170-012-4328-4.

[6] González-Neira, E.M., Montoya-Torres, J.R., Barrera, D. (2017). Flow-shop scheduling problem under uncertainties: Review and trends, International Journal of Industrial Engineering Computations, Vol. 8, No. 4, 399426, doi: 10.5267/j.ijiec.2017.2.001.

[7] Liu, Q., Ullah, S., Zhang, C. (2011). An improved genetic algorithm for robust permutation flowshop scheduling, The International Journal of Advanced Manufacturing Technology, Vol. 56, No. 1-4, 345-354, doi: $10.1007 /$ s00170-010-3149-6.

[8] Vallada, E., Ruiz, R., Minella, G. (2008). Minimising total tardiness in the $m$-machine flowshop problem: A review and evaluation of heuristics and metaheuristics, Computers \& Operations Research, Vol. 35, No. 4, 1350-1373, doi: $\underline{10.1016 / \text { i.cor.2006.08.016. }}$. 
[9] Yenisey, M.M., Yagmahan, B. (2014). Multi-objective permutation flow shop scheduling problem: Literature review, classification and current trends, Omega, Vol. 45, 119-135, doi: 10.1016/i.omega.2013.07.004.

[10] Sabuncuoglu, I., Goren, S. (2009). Hedging production schedules against uncertainty in manufacturing environment with a review of robustness and stability research, International Journal of Computer Integrated Manufacturing, Vol. 22, No. 2, 138-157, doi: 10.1080/09511920802209033.

[11] Yang, J., Yu, G. (2002). On the robust single machine scheduling problem, Journal of Combinatorial Optimization, Vol. 6, No. 1, 17-33, doi: 10.1023/A:1013333232691.

[12] Liu, L., Gu, H.-Y., Xi, Y.-G. (2007). Robust and stable scheduling of a single machine with random machine breakdowns, The International Journal of Advanced Manufacturing Technology, Vol. 31, No. 7-8, 645-654, doi: 10.1007/s00170-005-0237-0.

[13] Goren, S., Sabuncuoglu, I. (2008). Robustness and stability measures for scheduling: Single-machine environment, IIE Transactions, Vol. 40, No. 1, 66-83, doi: 10.1080/07408170701283198.

[14] Daniels, R.L., Carrillo, J.E. (1997). $\beta$-Robust scheduling for single-machine systems with uncertain processing times, IIE transactions, Vol. 29, No. 11, 977-985, doi: 10.1080/07408179708966416.

[15] Kuo, C.-Y., Lin, F.-J. (2002). Relative robustness for single-machine scheduling problem with processing time uncertainty, Journal of the Chinese Institute of Industrial Engineers, Vol. 19, No. 5, 59-67, doi: 10.1080/ 10170660209509359.

[16] Jia, Z., Ierapetritou, M.G. (2007). Generate Pareto optimal solutions of scheduling problems using normal boundary intersection technique, Computers \& Chemical Engineering, Vol. 31, No. 4, 268-280, doi: 10.1016/i.compchemeng.2006.07.001.

[17] Wu, C.W., Brown, K.N., Beck, J.C. (2009). Scheduling with uncertain durations: Modeling $\beta$-robust scheduling with constraints, Computers \& Operations Research, Vol. 36, No. 8, 2348-2356, doi: 10.1016/i.cor.2008.08.008.

[18] Fazayeli, M., Aleagha, M.-R., Bashirzadeh, R., Shafaei, R. (2016). A hybrid meta-heuristic algorithm for flowshop robust scheduling under machine breakdown uncertainty, International Journal of Computer Integrated Manufacturing, Vol. 29, No. 7, 709-719, doi: 10.1080/0951192X.2015.1067907.

[19] Gholami-Zanjani, S.M., Hakimifar, M., Nazemi, N., Jolai, F. (2017). Robust and fuzzy optimisation models for a flow shop scheduling problem with sequence dependent setup times: A real case study on a PCB assembly company, International Journal of Computer Integrated Manufacturing, Vol. 30, No. 6, 552-563, doi: 10.1080/ 0951192X.2016.1187293.

[20] Rahmani, D. (2017). A new proactive-reactive approach to hedge against uncertain processing times and unexpected machine failures in the two-machine flow shop scheduling problems, Scientia Iranica, Vol. 24, No. 3, 1571-1584, doi: 10.24200/sci.2017.4136.

[21] Cui, W., Lu, Z., Li, C., Han, X. (2018). A proactive approach to solve integrated production scheduling and maintenance planning problem in flow shops, Computers \& Industrial Engineering, Vol. 115, 342-353, doi: 10.1016/j.cie.2017.11.020.

[22] Ma, S., Wang, Y., Li, M. (2019). A novel artificial bee colony algorithm for robust permutation flowshop scheduling, In: Li, X., Wong, K.C. (eds.), Natural computing for unsupervised learning, Unsupervised and semisupervised learning, Springer, Cham, Switzerland,163-182, doi: 10.1007/978-3-319-98566-4_8.

[23] González-Neira, E.M., Urrego-Torres, A.M., Cruz-Riveros, A.M., Henao-García, C., Montoya-Torres, J.R., MolinaSánchez, L.P., Jiménez, J.-F. (2019). Robust solutions in multi-objective stochastic permutation flow shop problem, Computers \& Industrial Engineering, Vol. 137, Article No.106026, doi: 10.1016/j.cie.2019.106026.

[24] Lebbar, G., El Abbassi, I., Jabri, A., El Barkany, A., Darcherif, M. (2018). Multi-criteria blocking flow shop scheduling problems: Formulation and performance analysis, Advances in Production Engineering \& Management, Vol. 13, No. 2, 136-146, doi: 10.14743/apem2018.2.279.

[25] Xu, H., Bao, Z.R., Zhang, T. (2017). Solving dual flexible job-shop scheduling problem using a Bat Algorithm, Advances in Production Engineering \& Management, Vol. 12, No. 1, 5-16, doi: 10.14743/apem2017.1.235.

[26] Rathinam, B., Govindan, K., Neelakandan, B., Raghavan, S.S. (2015). Rule based heuristic approach for minimizing total flow time in permutation flow shop scheduling, Tehnički Vjesnik - Technical Gazette, Vol. 22, No. 1, 25-32, doi: $10.17559 /$ TV-20130704132725.

[27] Ma, D.Y., He, C.H., Wang, S.Q. Han, X.M., Shi, X.H. (2018). Solving fuzzy flexible job shop scheduling problem based on fuzzy satisfaction rate and differential evolution, Advances in Production Engineering \& Management, Vol. 13, No. 1, 44-56, doi: 10.14743/apem2018.1.272.

[28] Maqsood, S., Noor, S., Khan, M.K., Wood, A. (2012). Hybrid genetic algorithm (GA) for job shop scheduling problems and its sensitivity analysis, International Journal of Intelligent Systems Technologies and Applications, Vol. 11, No. 1-2, 49-62, doi: 10.1504/IJISTA.2012.046543.

[29] Khalid, Q.S,, Arshad, M., Maqsood, S., Jahanzaib, M., Babar, A.R., Khan, I., Mumtaz, J., Kim, S. (2019). Hybrid particle swarm algorithm for products' scheduling problem in cellular manufacturing system, Symmetry, Vol. 11, No. 6, 729, doi: 10.3390/sym11060729.

[30] Sarkar, B., Omair, M., Choi, S.-B. (2018). A multi-objective optimization of energy, economic, and carbon emission in a production model under sustainable supply chain management, Applied Sciences, Vol. 8, No. 10, 1744, doi: $10.3390 /$ app8101744.

[31] Zubair, M., Maqsood, S., Omair, M., Noor, I. (2019). Optimization of material handling system through material handling equipment selection, International Journal of Progressive Sciences and Technologies, Vol. 15, No. 2, 235243.

[32] Hayter, A. (2012). Instructor solution manual, Probability and statistics for engineers and scientists, $3^{\text {rd }}$ edition, Nelson Education, Scarborough, Canada.

[33] Rechenberg, I. (1973). Optimierung technischer systeme nach prinzipien der biologischen evolution, Phd thesis, Technische Universität Berlin, Germany, doi: 10.1002/fedr.19750860506. 
[34] Beyer, H.-G. (2001). The theory of evolution strategies, Natural Computing Series, Springer-Verlag, Berlin, Germany, doi: 10.1007/978-3-662-04378-3.

[35] Ahmad, A.M., Khan, G.M., Mahmud, S.A. (2013). Classification of arrhythmia types using cartesian genetic programming evolved artificial neural networks, In: Iliadis, L., Papadopoulos, H., Jayne, C. (eds.), Engineering applications of neural networks, EANN 2013, Communications in computer and information science, Vol. 383, Springer, Berlin, Germany, doi: 10.1007/978-3-642-41013-0 29.

[36] Paris, P.C.D., Pedrino, E.C., Nicoletti, M.C. (2015). Automatic learning of image filters using Cartesian genetic programming, Integrated Computer-Aided Engineering, Vol. 22, No. 2, 135-151, doi: 10.3233/ICA-150482.

[37] Murata, T., Ishibuchi, H., Tanaka, H. (1996). Genetic algorithms for flowshop scheduling problems, Computers \& Industrial Engineering, Vol. 30, No. 4, 1061-1071, doi: 10.1016/0360-8352(96)00053-8.

[38] Grabowski, J., Pempera, J. (2007). The permutation flow shop problem with blocking. A tabu search approach, Omega, Vol. 35, No. 3, 302-311, doi: 10.1016/i.omega.2005.07.004

[39] Nawaz, M., Enscore Jr., E.E., Ham, I. (1983). A heuristic algorithm for the m-machine, n-job flow-shop sequencing problem, Omega, Vol. 11, No. 1, 91-95, doi: 10.1016/0305-0483(83)90088-9.

[40] Carlier, J. (1978). Ordonnancements à contraintes disjonctives, RAIRO-Operations Research, Vol. 12, No. 4, 333 350, doi: 10.1051/ro/1978120403331.

[41] Reeves, C.R. (1995). A genetic algorithm for flowshop sequencing, Computers \& Operations Research, Vol. 22, No. 1, 5-13, doi: 10.1016/0305-0548(93)E0014-K. 


\title{
APEM
}

\section{Pristop s hibridno evolucijsko strategijo za robustno permutacijsko terminiranje velike proizvodnje po naročilu}

\author{
Khurshid, B. ${ }^{a}$, Maqsood, S. ${ }^{\text {b. }}$, Omair, M. ${ }^{b}$, Nawaz, R. ${ }^{a}$, Akhtar, R. ${ }^{a}$ \\ ${ }^{a}$ Department of Industrial Engineering, University of Engineering and Technology, Peshawar, Pakistan \\ ${ }^{b}$ Department of Industrial Engineering, Jalozai Campus, University of Engineering and Technology, Peshawar, Pakistan
}

\section{POVZETEK}

V tem prispevku je predlagan robusten razpored za obravnavo negotovosti pri permutacijah $m$-strojev za problem terminiranja velike proizvodnje po naročilu. Robusten razpored zagotavlja, da je pričakovani čas zaključka vedno krajši od časa izdelave. Za uporabo sposobnosti globalnega iskanja evolucijske strategije (ES) in sposobnosti lokalnega iskanja Tabu Search (TS), je za ustvarjanje zanesljivih razporedov z združevanjem izboljšanega ES s TS predlagana hibridna evolucijska strategija (HES). Robusten razpored smo najprej ustvarili z uporabo ES, nato pa smo rešitev optimizirali s pomočjo TS, da bi dobili največjo izkoriščenost in raziskanost prostora rešitev. Za največjo izkoriščenost smo v ES uporabili $(1+9)$ reprodukcijski operator in dvojno mutacijo. Za izboljšanje rezultatov pa smo uporabili tudi spremenljivo hitrost mutacije. V TS je bila dolžina tabu seznama nespremenljiva, za prihranek računskega časa pa se je uporabila spodnja meja. Hibridni algoritem je bil preizkušen na testnih primerih Carlierja in Reevesa iz knjižnice OR. Dosežene rezultate smo primerjali z drugimi znanimi tehnikami, ki so na voljo v literaturi. Rezultati so pokazali, da HES deluje bolje kot druge tehnike in zagotavlja povečanje verjetnosti, da je pričakovani čas zaključka krajši od časa izdelave.

(C) 2020 CPE, University of Maribor. All rights reserved.

\author{
PODATKI O ČLANKU \\ Ključne besede: \\ Permutacijsko terminiranje velike \\ proizvodnje po naročilu; \\ Razporejanje; \\ Carlier problem; \\ Reeves problem; \\ Evolucijsko računanje; \\ Hibridna evolucijska strategija; \\ Izboljšana evolucijska strategija; \\ Tabu iskanje \\ *Kontaktna oseba: \\ smaqsood@uetpeshawar.edu.pk \\ (Maqsood, S.) \\ Zgodovina članka: \\ Prejet 1. aprila 2020 \\ Popravljen 12. julija 2020 \\ Sprejet 14. julija 2020
}

